Apidologie, 1986, 17 (3), 257-266

\title{
UNTERSCHEIDUNG ZWISCHEN BIENEN VERSCHIEDENEN ALTERS DURCH VARROA JACOBSONI OUD. UND BEVORZUGUNG VON AMMENBIENEN IM SOMMERBIENENVOLK
}

\author{
Bernhard KRAUS, Nikolaus KOENIGER und Stefan FUCHS \\ Institut für Bienenkunde (Polytechnische Gesellschatt) \\ Fachbereich Biologie der J.W. Goethe-Universität \\ Karl-von-Frisch-Weg 2, D-6370 Oberursel/Ts.
}

\section{ZUSAMMENFASSUNG}

In Simultanwahlversuchen konnte gezeigt werden, daß die parasitische Milbe Varroa jacobsoni verschiedene Altersklassen von Bienenarbeiterinnen unterscheiden kann. Junge Ammenbienen (14 d) wurden gegenüber älteren Stockbienen und Flugbienen bevorzugt. Frischgeschlüpfte Bienen waren während der ersten 24 Stunden nur wenig attraktiv. Ahnliche Ergebnisse bei Verwendung durch Einfrieren abgetöteter Bienen legen die Beteiligung chemischer Faktoren an der Wahrnehmung des Bienenalters durch Varroa nahe.

Unterschiede ergaben sich auch hinsichtlich der Position der Milben auf dem Bienenkörper. Bei Ammenbienen befand sich Varroa häufiger unter den Abdominalsterniten als bei älteren Bienen, bei denen sie zu einem höheren Prozentsatz zwischen Thorax und Abdomen gefunden wurde.

Auch unter natürlichen Bedingungen im Bienenvolk konnte ein höherer Befall von Ammenbienen nachgewiesen werden. Die Ergebnisse zeigen, daß Varroa in der Lage ist, eine aktive Auswahl zwischen den Wirtsbiene zu treffen.

\section{EINLEITUNG}

Der Lebenszyklus von $V$. jacobsoni umfasst eine Reproduktionsphase in den verdeckelten Brutzellen und eine Überdauerungs- und Infektionsphase auf den Adultbienen im Bienenvolk. Während die Milbe auf den Bienen parasitiert, könnten ihre Überlebens- und Reproduktionschancen auf mehrere Weise durch altersabhängige Unterschiede zwischen den Arbeiterinnen beeinflußt werden. Jüngere Stockbienen könnten vorteilhaft als Transportmittel zu den Brutzellen dienen, da sie häufig in Kontakt zu den Larven kommen oder bei der Zellenverdeckelung mitwirken. Der höhere Juvenilhormontiter (JH-III) in der Hämolymphe von älteren Arbeiterinnen verstärkt nach Untersuchungen von HäNEL 
(1983, 1986) die Neigung des Parasiten, Zellen aufzusuchen und mit der Reproduktion zu beginnen. Der Aufenthalt auf Flugbienen könnte einerseits die Verbreitung von Volk zu Volk begünstigen, birgt aber anderseits das Risiko, mit den Bienen außerhalb des Volkes umzukommen.

Auf diese Weise besteht die Möglichkeit, daß Varroa durch das Aufsuchen der jeweils geeigneten Wirtsbiene ihr Reproduktions- bzw. Verbreitungsverhalten aktiv steuert. Eine notwendige Vorraussetzung hierfür ist die Fähigkeit des Parasiten, Arbeiterinnen verschiedener Alters- oder Tätigkeitsgruppen voneinander unterschieden zu können. Dies sollte in der vorliegenden Arbeit durch Simultanwahlversuche geklärt werden. Aus der Untersuchung der Befallsdichte von Altersgruppen der Arbeiterinnen im Bienenvolk sollten zusätzliche Hinweise auf eine Altersgruppenbevorzugung unter natürlichen Bedingungen gesammelt werden.

\section{MATERIAL UND METHODE}

Die Untersuchungen wurden an Apis mellifera carnica Pollmann von Juni bis September 1984 durchgeführt. Bei den Simultanwahlversuchen konnte jeweils eine Varroa zwischen 3 unterschiedlich alten oder verschieden aufgezogenen Bienen wählen. 0-24 $\mathrm{h}$ alte Jungbienen wurden von im Brutschrank geschlüpften Brutwaben abgesammelt. 4-6 Tage alte altersmarkierte Ammenbienen entstammten einem Versuchsvolk, als Flugbienen wurden Pollensammlerinnen am Flugloch abgesammelt.

Die Versuchsarena bestand aus Polystyrolpetrischalen $60 \times 15 \mathrm{~mm}$, deren Boden mit Wachs ausgegossen war. Durch eine Öffnung im Deckel der Petrischale konnte die Versuchsmilbe an einem feuchten Pinsel hängend in die Mitte der Arena gesetzt werden. Bei länger dauernden Versuchen wurden die Bienen mit Futterteigkugeln gefüttert. Bei Versuchen mit toten Bienen lagen die Tiere in gleichem Abstand voneinander und mit zum Petrischaleninneren gerichteter Dorsalseite am Schalenrand. Die Temperatur entsprach der Raumtemperatur von etwa $20-25^{\circ} \mathrm{C}$.

Für Versuche im Volk wurden im Abstand von 6 Tagen jeweils mehrere hundert frischgeschlüpfte Bienen mit in Schellack eingerührten Farbpigmenten auf dem Thorax markiert und in das Versuchsvolk eingebracht. Alle 5-6 Tage wurden die wiederauffindbaren markierten Arbeiterinnen abgefangen und der Befall mit Varroa sowie die Position der Milben auf dem Bienenkörper registriert. Zusätzlich wurden heimkehrende Pollensammlerinnen auf Varroabefall hin untersucht.

\section{ERGEBNISSE}

1) Verteilung und Position von $V$. jacobsoni auf Bienen verschiedenen Alters in Simultanwahlversuchen

Die Präferenzuntersuchungen an Varroa bei 147 Wahlversuchen mit lebenden Bienen (Abb. 1) zeigten 2 Stunden nach Versuchsbeginn einen deutlich niedrigeren 


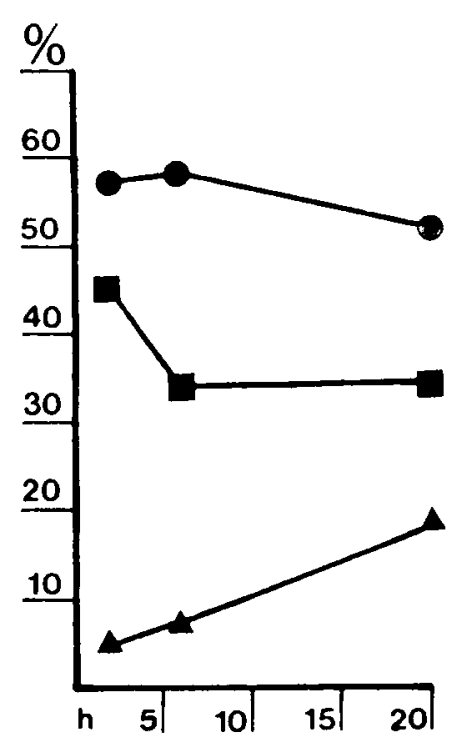

Aвв. 1. - Verteilung von Varroa in Simultanwahlversuchen zwischen Jungbienen (24 h, A), Ammen (14 d, -) und Pollensammlerinnen ( $\mathbf{0})$

Abszisse : Stunden nach Versuchsbeginn (h). Ordinate : Anteil von Varroa $(\%, \mathrm{~N}=147)$

FIG. 1. - Distribution of Varroa mites among young bees $(24 \mathrm{~h}, \mathbf{\Delta})$, nurses $(14 \mathrm{~d},-)$ and pollen collectors $(\square)$ in tests of simultaneous choice Abscissa : hours since test start (h). Ordinate : proportion of Varroa $(\%, N=147)$

Befall von Jungbienen $(J: 7)$ verglichen mit dem von Ammen $(A: 68)$ oder Pollensammlerinnen ( $P: 59$; keine Entscheidung : 13. Statistische Sicherung $: J \mathrm{zu}$ $A: \mathrm{Chi}^{2}=49.6, \mathrm{p}<0.001 ; J$ zu $\left.P: \mathrm{Chi}^{2}=41.0, \mathrm{p}<0.001\right)$. Die Verteilung der Milben zwischen Ammen und Pollensammlerinnen ist hierbei etwa gleich $\left(\mathrm{Chi}^{2}=0.6\right.$, n.s. $)$. Auch nach 6 Stunden lag der Besatz der Jungbienen klar unter dem der Ammen oder Pollensammlerinnen $(J: 9 ; A: 81 ; P: 47$. Statistische Sicherung $: J$ zu $A: \mathrm{Chi}^{2}=57.6, \mathrm{p}<0.001 ; J$ zu $\left.P: \mathrm{Chi}^{2}=25.8, \mathrm{p}<0.001\right)$. Pollensammlerinnen waren zu diesem Zeitpunkt jedoch deutlich weniger befallen als Ammenbienen $\left(\mathrm{Chi}^{2}=9.0, \mathrm{p}<0.01\right)$.

Besonders auffällig war hierbei die sehr geringe Attraktivität von frischgeschlüpften Jungbienen, die dazu führte, daß diese oft sogar dann von den Milben verlassen wurden, wenn keine anderen Bienen zur Auswahl standen. Für diese zeigte sich im Simultanwahlversuch ein deutlicher Attraktivitätsanstieg in den ersten 24 Stunden (100 Versuchsansätze. $0-1 \mathrm{~h}: 16$ Varroa; 4-5 h : 30 Varroa ; 23-24 h : 45 Varroa. Erste und letzte Gruppe : $\mathrm{Chi}^{2}=21.4$, p $<0.001$ ). 
Die Präferenzuntersuchungen mit toten Ammen, Jungbienen und Pollensammlerinnen (Abb. 2) umfassten 319 Versuchsansätze. 2 Stunden nach Versuchsbeginn waren Ammen häufiger befallen als Jungbienen oder Pollensammlerinnen $\left(A: 17 ; J: 34 ; P: 111\right.$. Statistische Sicherung : $A$ zu $J: \mathrm{Chi}^{2}=94.2, \mathrm{p}<0.001$; $A$ zu $\left.P: \mathrm{Chi}^{2}=13.9, \mathrm{p}<0.001\right)$ und Pollensammlerinnen ihrerseits häufiger befallen als Jungbienen $\left(P \mathrm{zu} J: \mathrm{Chi}^{2}=40.9, \mathrm{p}<0.001\right)$. Hinweise auf einen Einfluß der Herkunft der Milben oder des Milbenalters ergaben sich nicht. Weder die Position auf den Herkunftsbienen noch deren Alter noch die Färbung der Milbe beeinflußten das Ergebnis der Simultanwahlversuche deutlich.

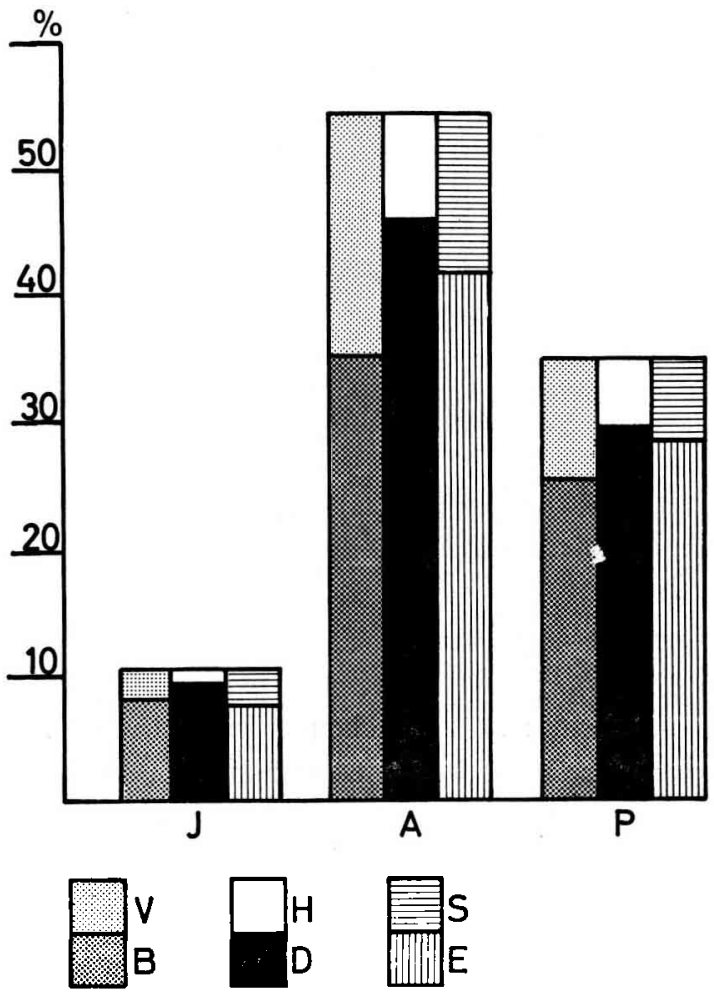

Aвв. 2. - Verteilung von Varroa

auf toten Bienen verschiedener Altersklassen 2 Stunden nach Versuchsbeginn

$\mathbf{J}=$ Jungbiene, $\mathbf{A}=$ Amme, $\mathbf{P}=$ Pollensammlerin. Ordinate : Anteil von Varroa $(\%, \mathbf{N}=319)$. Spezifikaton der Milben : Aus dem Volk (V) oder von Jungbienen aus dem Brutschrank (B); hell bis mittelbraum (H) oder dunkel (D);

vor dem Versuch unter Abdominalsternit (S) oder anderswo (E)

FIG. 2. - Distribution of Varroa mites on different age groups of dead bees after 2 hours

$\mathbf{J}=$ young bee, $\mathbf{A}=$ nurse, $\mathbf{P}=$ pollen collector. Ordinate : proportion of Varroa $(\%, \mathbf{N}=319)$.

Specification of mites : From hive (V) or from incubated young bees (B);

light or medium brown (H) or dark (D) ;

before test under abdominal sternites (S) or elsewhere (E) 
Bei der Untersuchung der Abhängigkeit der Position der Milbe auf dem Bienenkörper vom Alter des Wirtstieres wurden nur die mit lebenden Bienen durchgeführten Versuche in Betracht gezogen. Abb. 3 zeigt die von den Milben am häufigsten eingenommenen Positionen am Bienenkörper bei Trachtbienen und Ammenbienen 2, 6 und $20 \mathrm{~h}$ nach Versuchsbeginn zusammengefasst. Die Positionshäufigkeiten waren 2 und $6 \mathrm{~h}$ nach Versuchsbeginn signifikant verschieden $\left(2 \mathrm{~h}: \mathrm{Chi}^{2}=16.8\right.$, df $=5, \mathrm{p}<0.01 ; 6 \mathrm{~h}: \mathrm{Chi}^{2}=25.9$, df $\left.=5, \mathrm{p}<0.001\right)$. Besonders auffällig war ein $6 \mathrm{~h}$ nach Versuchsbeginn bei den Ammen deutlich höherer Anteil von Milben unter den Abdominalsterniten (Avs; 21 von 78; Pollensammlerinnen : 1 von $47, \mathrm{Chi}^{2}=10.1, \mathrm{p}<0.01$ ) und ein geringerer Anteil in der Position zwischen Thorax und Abdomen (TA; 5 von 78 ; Pollensammlerinnen : 17 von $47, \mathrm{Chi}^{2}=9.4, \mathrm{p}=0.01$ ).
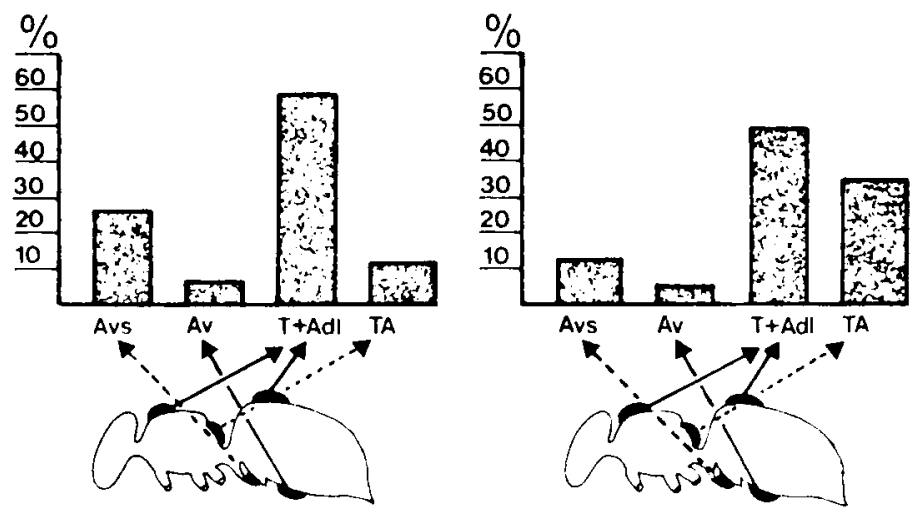

AвB. 3. - Relative Häufigkeiten der wichtigsien Positionen von Varroa auf dem Körper von Bienenarbeiterinnen (Ordinate, \%) im Arenaversuch

Linkes Diagramm : Ammenbienen $(\mathrm{N}=634)$, rechtes Diagramm : Pollensammlerinnen $(\mathrm{N}=432)$.

Positionen : Thorax (T), zwischen Thorax und Abdomen (TA),

Abdomen ventral (Av) unter Sterniten (Avs) oder dorsal/lateral (Adl)

FIG. 3. - Relative frequencies of the main positions of Varroa on worker bees during tests of simultaneous choice (Ordinate, \%)

Left diagram : nurses $(N=634)$; right diagram : pollen collectors $(N=432)$.

Positions : thorax (T), between thorax and abdomen (TA),

abdomen ventral (Av) under sternites (Avs) or dorsal/lateral (Adl)

Zur Überprüfung der Frage, ob die Präferenz für Ammenbienen auch unabhängig von deren Aufenthalt im Bienenvolk auftritt, wurden 4 Tage alte Stockbienen und 4 Tage alte im Käfig aufgezogene Bienen gegen Jungbienen getestet (Abb. 4). Die in Käfigen gehaltenen Bienen waren signifikant häufiger 
von Varroa besetzt als die Jungbienen ( 21 zu 5 Varroa, $\mathrm{Chi}^{2}=9.9, \mathrm{p}<0.01$ ), allerdings waren sie weniger stark befallen als die gleichalten Bienen aus dem Volk (21 zu $37 ; \mathrm{Chi}^{2}=4.4, \mathrm{p}<0.05$ ).

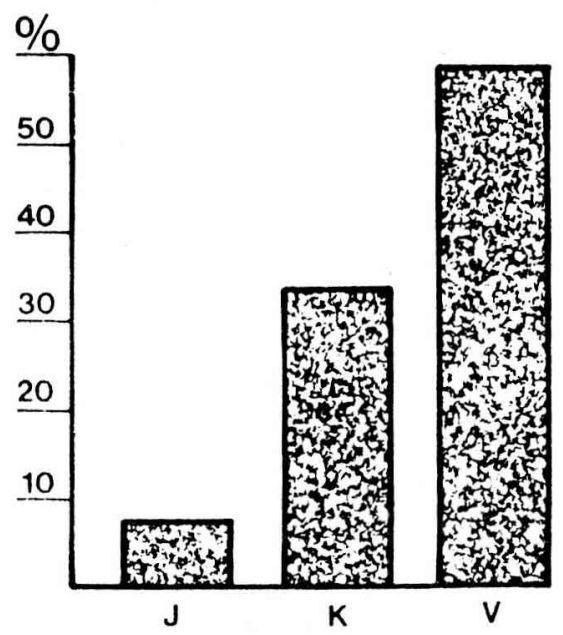

ABB 4. - Verteilung von Varroa (Ordinate, \%, $N=72$ ) zwischen Jungbienen (J) und 4 Tage alten Arbeiterinnen entweder aus dem Volk (V) oder in Käfigen separat gehalten (K) im Simultanwahlversuch nach 2 Stunden

Fig. 4. - Distribution after 2 hours during tests of simultaneous choice of Varroa mites (ordinate, $\%, N=72$ ) among young bees (J) and 4-day-old worker bees from a hive,

$(V)$ or kept seperate in cages $(K)$

2) Verteilung und Position von $V$. jacobsoni auf markierten Altersgruppen im Volk

Abb. 5 zeigt den relativen Varroabefall von 3 markierten Bienengruppen bei zunehmendem Bienenalter. Die Abnahme des relativen Bienenbefalls von den 12 Tage alten Bienen zu den 18 Tage alten Bienen ist signifikant (vgl. Tabl. 1; $\left.\mathrm{Chi}^{2}=5.4 ; \mathrm{p}<0.025\right)$. Eine bessere statistische Schireung erhält man bei Zusammenfassung der 6 und 12 Tage alten Bienen mit den 18 und 24 Tage alten Bienen $\left(\mathrm{Chi}^{2}=15.22 ; \mathrm{p}<0.001\right)$. Der relative Befall der Pollensammlerinnen ist niedriger als der der 6,12 oder 18 Tage alten Bienen $\left(\mathrm{Chi}^{2}=17.8\right.$, $\left.\mathrm{p}<0.001 ; \mathrm{Chi}^{2}=16.9, \mathrm{p}<0.001 ; \mathrm{Chi}^{2}=5.8, \mathrm{p}=0.025\right)$.

Die Untersuchung der Position des Parasiten auf dem Bienenkörper ergab bei 6 und 12 Tage alten Bienen einen größeren Anteil von Varroa unter den Abdominalsterniten (58 von 66 Varroa) als bei 18 und 24 Tage alten Bienen oder Pollensammlerinnen ( 9 von 17 Varroa; $\mathrm{Chi}^{2}=13.3, \mathrm{p}<0.001$ ). 


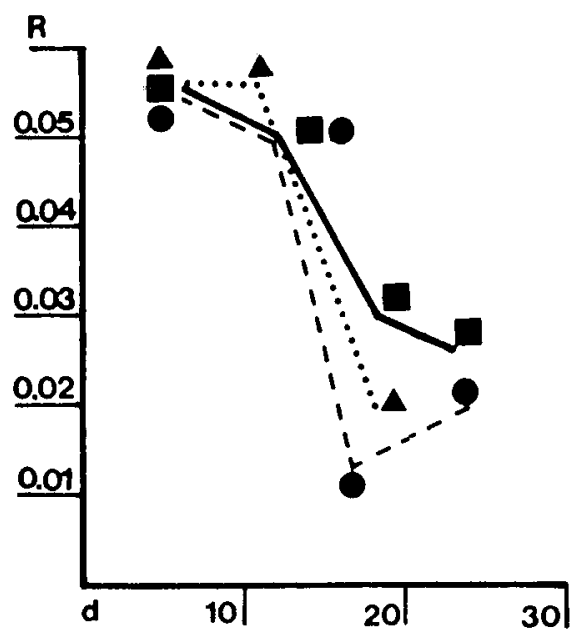

Aвв, 5. - Relativer Varroabefall (Ordinate; $R=$ Varroa/Biene)

in drei Bienengruppen aus dem Volk in Abhängigkeit vom Bienenalter (Abszisse).

Beginn der Serien : 21.7 (O), 27.8 (), 2.8 (A)

Fig. 5. - Relative infestation with Varroa mites (ordinate; $R=$ Varroa/bees) of three groups of worker bees in relation to the bee age (abscissa).

Start of the groups : $21.7(\bullet), 27.8(\mathbf{\square}), 2.8(\boldsymbol{A})$

TAB. 1. - Relativer Befall der Bienenaltersgruppen mit Varroa im Volk.

TABL. 1. - Infestation rates of age groups of worker bees within a hive.

\begin{tabular}{c|c|c|c}
\hline $\begin{array}{c}\text { Alter der Bienen } \\
\text { Age of bees }\end{array}$ & $\begin{array}{c}\text { Anzahl der Bienen } \\
\text { Number of bees }\end{array}$ & $\begin{array}{c}\text { Gefundene Varroa } \\
\text { Varroa found }\end{array}$ & $\begin{array}{c}\text { Relativer Befall } \\
\text { Infestation rate }\end{array}$ \\
\hline 6 & 600 & 33 & 0.055 \\
12 & 640 & 33 & 0.052 \\
18 & 450 & 10 & 0.022 \\
24 & 250 & 6 & 0.032 \\
Ps & 370 & 1 & 0.003 \\
\hline
\end{tabular}

Ps : Pollensammlerinnen.

Pollen collectors.

\section{DISKUSSION}

Grobov (1976), ToschKoff et al. (1979) und RitTer (1981) erwähnen einen besonders hohen Befall der Ammenbienen, ohne dies aber näher zu belegen. Die Untersuchung des Bienenbefalls im Volk in Abhängigkeit vom Bienenalter bestätigt diese Ansicht zumindest für die Sommermonate. 
In Simultanwahlversuchen konnte nachgewiesen werden, daß Varroa das Alter von Bienenarbeiterinnen unterscheiden kann. Hierbei zeigte sich eine auffällig geringe Attraktivität frisch geschlüpfter Bienen. Diese nimmt bereits während der ersten 24 Stunden deutlich zu, um noch während der Stockbienenphase wieder deutlich zu sinken. HüTtINGER et al. (1980) fanden übereinstimmend einen besonders niedrigen Befall bei Wassersammlerinnen, sodaß die sehr geringe Attraktivität von Pollensammlerinnen für Flugbienen allgemein gelten dürfte. Die Ergebnisse mit außerhalb des Volks gehaltenen Arbeiterinnen zeigen, daß die Attraktivität der Ammen nicht ausschließlich auf einem Stockfaktor beruht, etwa in der Form eines auf die Milben anziehend wirkenden, beim Füttern angenommenen Brutgeruchs. Die Attraktivität lag allerdings deutlich unter der von gleichaltrigen Stockbienen. Die Ähnlichkeit der Versuchsergebnisse mit durch Einfrieren abgetöteten Bienen legt die Beteiligung chemischer Faktoren nahe. Daß Chemorezepiton bei der Orientierung des Parasiten eine Rolle spielen kann, konnte von HOPPE und SCHLEY (1984) wahrscheinlich gemacht werden.

Die Bedeutung der unterschiedlichen Positionen von Varroa auf den Bienen verschiedenen Alters sind bisher nicht sicher $\mathrm{zu}$ deuten. Die Position zwischen Thorax und Abdomen sowie zwischen Kopf und Thorax erscheinen besonders geschützt und könnten eine sichere Transportposition besonders während des Flugs der Biene darstellen, während die Positionen direkt auf Thorax oder Abdomen einen bevorstehenden Wechsel des Wirtstieres anzudeuten scheinen. Es ist sehr wahrscheinlich, daß die Position zwischen den Abdominalsterniten bevorzugte Position zur Nahrungsaufnahme ist und daß damit von den Milben überwiegend Hämolymphe von Jungbienen aufgenommen wird. Eine genauere Untersuchung der unterschiedlichen Positionen von Varroa auf den Wirtsbienen und deren Bedeutung fehlt bisher und wäre insbesondere in Hinblick auf einen Zusammenhang mit dem Juvenilhormontiter der Bienen, dem Befallsgrad der Bienenvölker oder der Jahreszeit sehr wünschenswert.

Sowohl das Ergebnis einer Präferenz für nur bis ca. 2 Wochen alte Arbeiterinnen als auch die Unterschiedlichkeit der Vorzugspositionen auf dem Bienenkörper sprechen dafür, daß die Phase außerhalb der Zellen in der Biologie des Parasiten eine wichtige Rolle spielt. Die Ergebnisse von HäNEL (1983, 1986) hatten gezeigt, daß Varroa an seinen Wirt hormonell gekoppelt ist. Im Vergleich zu auf jüngeren Bienen parasitierenden Milben drangen Varroa von älteren Flugbienen, die einen höheren Titer von JH-(III) aufweisen, zu höheren Prozentsätzen in Zellen ein und zeigten durch die Sekretfüllung einer Zellgruppe des Neurosekretorischen Systems die beginnende Eilage an.

Nach den vorliegenden Ergebnisse wären hiernach die Vorraussetzungen gegeben, daß eine Regulierung des Reproduktionsverhaltens durch die Hämolymphaufnahme nicht nur rein passiv durch die zufällige Verteilung der Milben auf die 
Altersgruppen, sondern durch aktive Auswahl des Wirtstieres durch die Milben erfolgen kann. Die Ergebnisse sprechen aber eindeutig gegen die Vermutung einer bevorzugten Hämolymphaufnahme an den älteren Stockbienen und den Flugbienen. Eine weitere Denkmöglichkeit ist, daß die Milben Flugbienen erst besteigen, sobald sie durch Nahrungsaufnahme an jüngeren Bienen für die Reproduktion genügend Reservestoffe angesammelt haben. Während des kurzen Aufenthalts auf Flugbienen wären sie dann dem Risiko ausgesetzt, mit diesen im Freien umzukommen. Sie könnten dann aber entweder im eigenen Volk eine Zelle zur Reproduktion aufsuchen oder zu einem sehr geringen Prozensatz durch Verflug zur Neuinfektion benachbarter Völker beitragen. Die Klärung dieser und ähnlicher Fragen erfordert die Rekonstruktion des Lebenszyclus von Varroa während der Zeit außerhalb der Zellen, zu der bislang noch viele Einzelheiten fehlen.

Mit Unterstützung der Deutschen Forschungsgemeinschaft (Ko 400/4-1).

Eingegangen im Februar 1986. Angenommen im Juni 1986.

\author{
SUMMARY \\ RECOGNITION AND PREFERENCE OF BEES OF SPECIFIC AGE \\ BY VARROA JACOBSONI
}

\begin{abstract}
Test of simultaneous choice demonstrated the capability of Varroa mites to differentiate among bees of different ages and confirmed a preference for nurses. After 6 hours of the test runs about $60 \%$ of the tested mites were situated on nurses, $35 \%$ on pollen collectors but only $5 \%$ on young bees which had just been uncapped. Tests using dead bees killed by freezing indicated the involvement of chemical factors. In these tests $55 \%$ of the mites were found on nurses, $35 \%$ on pollen collectors and $10 \%$ on young bees.

The relative infestation of bees of different ages within the hive were determined by investigating, the numbers of mites on marked age groups. The results showed about a 2-3 fold higher infestation of young bees (14 d) compared to older bees from the hive and about a 20 fold higher infestation compared to pollen collectors.

Differences were also found in the positions of the mites on the bees. During the tests of simultaneous choice about $35 \%$ of the mites on pollen collectors were positioned between the thorax and abdomen and $12 \%$ under the abdominal sternites, whereas in nurses only $11 \%$ were between the thorax and abdomen but $26 \%$ were under the abdominal sternites. The results indicate an active choice of its host bee by Varroa, which might be of some influence on its life cycle.
\end{abstract}




\section{RESUME}

\section{RECONNAISSANCE PAR VARROA JACOBSONI OUD. DES ABEILLES D'AGE DIVERS ET PREFERENCE POUR LES NOURRICES}

Des tests de choix simultané ont montré la capacité des acariens Varroa à reconnaître des abeilles de différents âges et ont confirmé leur préférence pour les nourrices. Au bout de 6 heure: de test, environ $60 \%$ des acariens testés se trouvent sur les nourrices, $35 \%$ sur les butineuses de pollen et seulement $5 \%$ sur les jeunes abeilles fraîchement écloses. Les tests utilisant des abeilles mortes tuées par congélation indiquent que des facteurs chimiques interviennent. Dans ces tests $55 \%$ des acariens sont trouvés sur les nourrices, $35 \%$ sur les butineuses de pollen et $10 \%$ sur les jeunes abeilles.

L'infestation relative des abeilles selon leur âge à l'intérieur de la colonie a été déterminée d'après le nombre d'acariens présents sur des groupes d'âge connu et marqué. Les résultats montrent une infestation 2 à 3 fois plus forte chez les jeunes abeilles (14 jours) comparée aux plus vieilles et environ 20 fois plus forte comparée aux butineuses de pollen.

On a trouvé également des différences concernant la position des acariens sur les abeilles. $\mathrm{Au}$ cours des tests de choix simultané environ $35 \%$ des acariens présents sur les butineuses de pollen étaient situés entre le thorax et l'abdomen et $12 \%$ sous les sternites abdominaux, tandis que chez les nourrices seulement $11 \%$ étaient entre le thorax et l'abdomen, mais $26 \%$ sous les sternites abdominaux. Les résultats montrent un choix actif de l'abeille hôte par Varroa jacobsoni, choix susceptible d'avoir une influence sur son cycle de développement.

\section{LITERATUR}

Grobov O.F., 1977. - Varroasis in bees. In : Varroasis a honeybee disease. Apimondia Publishing House, Bukarest, 46-70.

HäNEL H., 1983. - Effect of JH-(III) on the reproduction of Varroa jacobsoni. Apidologie, 14 (2), 137-142.

Hänel H., Koeniger N., 1986. - Possible regulation of the reproduction of the honeybee mite Varroa jacobsoni (Merostigmata, Acari) by a host's hormone : Juvenile hormone (III). J. Insect. Physiol. (in press).

Hoppe H., Schley P., 1984. - Varroa-Falle mit Lockstoffen möglich ? Die Biene, 9, 187-188.

Hüttinger E., Pechhacker H., Sulimanovic D., Tomac I., 1980. - Zur Ausbreitung von Varroa jacobsoni Oud. von Volk zu Volk. In : Diagnose und Therapie der Varroatose. ApimondiaVerlag, Bukarest, 56-62.

RITter W., 1981. - Varroa disease of the honey bee Apis mellifera. Bee World, 62 (4), 141-153.

Toschкoff A., 1979. - Neues im Studium und der Bekämpfung der Varroatose. In : Behämpfung und Vorbeugung der Varroatose. Apimondia-Verlag, Bukarest, 32-35. 